\title{
Comparative Analysis of Immunoinflammatory and Nutritional Measures in Surgically Resected Esophageal Cancer: A Single-center Retrospective Study
}

\author{
MAKOTO SAKAI ${ }^{1}$, MAKOTO SOHDA ${ }^{1}$, HIDEYUKI SAITO ${ }^{1}$, YASUNARI UBUKATA ${ }^{1}$, \\ NOBUHIRO NAKAZAWA ${ }^{1}$, KENGO KURIYAMA ${ }^{1}$, KEIGO HARA ${ }^{1}$, AKIHIKO SANO ${ }^{1}$, \\ KYOICHI OGATA $^{1}$, TAKEHIKO YOKOBORI ${ }^{2}$, KEN SHIRABE $^{3}$ and HIROSHI SAEKI ${ }^{1}$ \\ ${ }^{1}$ Division of Gastroenterological Surgery, Department of General Surgical Science, \\ Graduate School of Medicine Gunma University, Gunma, Japan; \\ ${ }^{2}$ Initiative for Advance Research, Gunma University, Gunma, Japan, \\ ${ }^{3}$ Department of General Surgical Science, Gunma University, \\ Graduate School of Medicine, Gunma, Japan
}

\begin{abstract}
Background/Aim: Several immunoinflammatory and nutritional measures have been reported to be good prognostic indicators for esophageal cancer (EC). However, the association between those markers and the postoperative survival of EC patients remains unclear due to varying study designs and treatment strategies. The aim of this study was to compare the significance of preoperative immunoinflammatory and nutritional measures in patients with EC. Patients and Methods: One hundred and five patients with EC who underwent McKeown esophagectomy with gastric tube reconstruction without neoadjuvant therapy between 2006 and 2014 were included in this study. The prognostic values of preoperative modified Glasgow prognostic score (mGPS), controlling nutritional status (CONUT) score, prognostic nutritional index (PNI), $C$-reactive protein $(C R P)$-to-albumin ratio $(C A R)$, neutrophil-to-lymphocyte ratio (NLR), and platelet-to-lymphocyte ratio $(P L R)$ were investigated using univariate and multivariate survival analyses. Results: Multivariate analysis revealed that $C A R$ and pathological stage are independent prognostic factors for overall survival (OS). CAR was significantly associated with more advanced pathological stage as both a subject and a continuous
\end{abstract}

This article is freely accessible online.

Correspondence to: Makoto Sohda, Department of General Surgical Science, Gunma University, Graduate School of Medicine, 3-39-22 Showa-machi, Maebashi, Gunma 371-8511, Japan. Tel: +81 272208224, Fax: +81 272208230, e-mail: msohda@ gunma-u.ac.jp

Key Words: Esophageal Neoplasms, C-reactive protein, albumin, nutrition, inflammation. variable. Conclusion: Preoperative CAR was an independent prognostic factor for the OS of EC patients who underwent McKeown esophagectomy. The tumor-stage related increase in CAR demonstrated that a high CAR is associated with tumor progression in EC patients.

Systemic inflammation and malnutrition have received increasing attention in many malignancies because they are reported to be associated with a poor prognosis. To date, several immunoinflammatory and nutritional measures have been reported to be good prognostic indicators for esophageal cancer (EC). The modified Glasgow prognostic score (mGPS), an inflammation-based prognostic score consisting of serum C-reactive protein (CRP) and albumin, has been reported as an independent prognostic factor in early EC patients (1). The controlling nutritional status (CONUT) score, consisting of serum albumin, cholesterol, and total lymphocyte count, has been reported to be associated with the survival of EC patients undergoing surgery (2). The prognostic nutritional index (PNI) is calculated using serum albumin levels and the total lymphocyte count, and has been reported to be correlated with the incidence of postoperative complications and prognosis of patients with EC (3). The C-reactive protein (CRP)-to-albumin ratio (CAR) has been found to have prognostic value in EC patients (4-7). A high neutrophil-tolymphocyte ratio (NLR) has been reported to be associated with poor overall survival (OS) and it may be a significant predictive biomarker in EC patients (8). A high platelet-tolymphocyte ratio (PLR) has also been demonstrated to be a significant predictor of OS in EC patients (9). However, the association between these markers and the postoperative survival of EC patients remains unclear due to varying study 


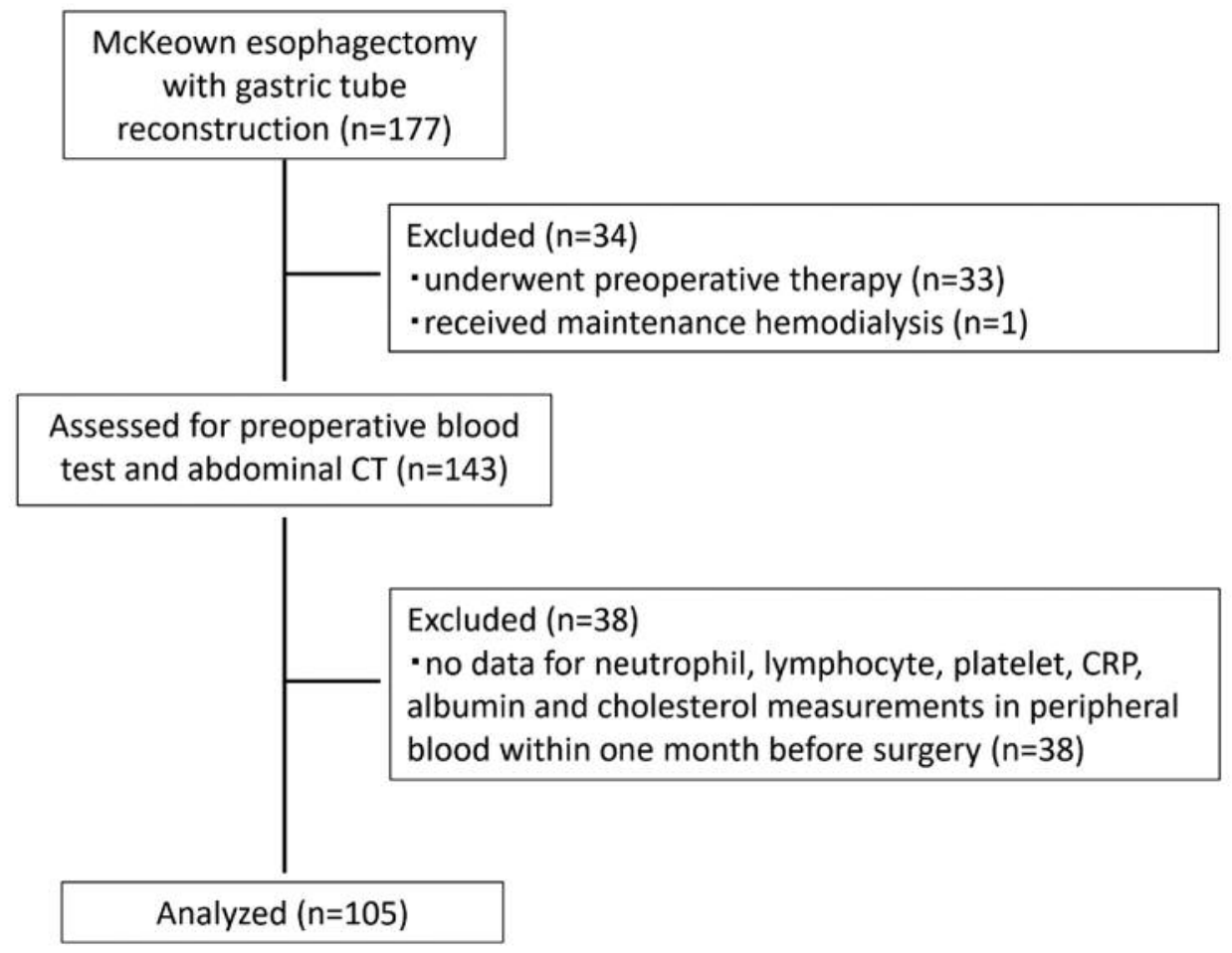

Figure 1. Flowchart showing patient recruitment.

designs, such as sample size and eligibility criteria, and treatment strategies, such as with or without neoadjuvant therapy and surgical procedure. We hypothesized that the association between these markers and EC survival will be clearer when the heterogeneity concerning the patient background and the timing of sample collection is minimal. To investigate this hypothesis, we examined the prognostic value of mGPS, CONUT, PNI, CAR, NLR, and PLR in EC patients who underwent McKeown esophagectomy with gastric tube reconstruction without neoadjuvant therapy.

\section{Patients and Methods}

Patients. This retrospective study was approved by the ethics committee of the Graduate School of Medicine, Gunma University (Protocol number: HS2019-025). Informed consent was obtained in the form of opt-out on the web-site. The criterion for inclusion was thoracic EC patients who underwent radical McKeown esophagectomy with gastric tube reconstruction between 2006 and 2014 at our institute. Patients were excluded from this study if they had: i) an active infection, ii) had received maintenance hemodialysis, iii) had undergone preoperative therapy, or iv) had no data of neutrophil, lymphocyte, platelet, CRP, albumin, or cholesterol measurements in peripheral blood within one month before surgery. Hospital patient records were reviewed for tumor characteristics and patient outcomes. The tumor stage and disease grade were classified according to the 7th edition of the TNM classification of the International Union Against Cancer (UICC) (10). The tumor stage was determined conventionally using computed tomography (CT) of the neck, chest, and abdomen, endoscopic ultrasonography (EUS), endoscopy, and esophagography. Follow-up was generally performed at 3- to 4-month intervals within the first two years after surgery and every six months up to five years.

Measurement of prognostic scores. The mGPS was constructed as below. Patients with both a high C-reactive protein level ( $>10 \mathrm{mg} / \mathrm{l})$ and hypoalbuminemia $(<35 \mathrm{~g} / \mathrm{l})$ were allocated a score of 2 . Patients with only a high C-reactive protein level $(>10 \mathrm{mg} / \mathrm{l})$ were allocated a score of 1 . Patients without a high $\mathrm{C}$-reactive protein level were allocated a score of 0 (11). CONUT scores were calculated based on serum albumin levels, absolute lymphocyte count, and cholesterol levels (12), summarized as follows: i) albumin concentration at $\geq 3.5,3.0-3.49,2.5-2.99$, and $<2.5 \mathrm{~g} / \mathrm{dl}$ was scored as $0,2,4$, and 6 points, respectively, ii) total lymphocyte count at $\geq 1600,1200-1599,800-1199$, and $<800 / \mathrm{mm}^{3}$ was scored as $0,1,2$, and 3 points, respectively, iii) total cholesterol concentration at $\geq 180,140-179,100-139$, and $<100 \mathrm{mg} / \mathrm{dl}$ was scored as $0,1,2$, and 3 points, respectively (13). The CONUT score was defined based on the total scores for these points. PNI was calculated as $10 \times$ the serum albumin level $(\mathrm{g} / \mathrm{dl})+0.005 \times$ the absolute lymphocyte count (14).CAR was calculated as the ratio of serum CRP $(\mathrm{mg} / \mathrm{dl})$ to serum albumin (g/dl) (15). NLR was calculated as the absolute neutrophil count divided by the absolute lymphocyte count (4). PLR was calculated as the absolute platelet count divided by the absolute lymphocyte count (4).

Statistical analysis. Subject characteristics were compared using chi-squared tests for categorical variables and the Student's $t$-test 
Table I. Patient characteristics.

\begin{tabular}{llc}
\hline Characteristics & & No. of patients $(\%)$ \\
\hline Age (years), mean (SD) & & $64.75(8.73)$ \\
Gender & Male & $93(88.6)$ \\
Location of tumor & Female & $12(11.4)$ \\
& Upper & $11(10.5)$ \\
mGPS & Middle & $52(49.5)$ \\
& Lower & $42(40.0)$ \\
CONUT score & 0 & $91(86.7)$ \\
& 1 & $10(9.5)$ \\
& 2 & $4(3.8)$ \\
& 0 & $28(26.7)$ \\
PNI (mean (SD)) & 1 & $37(35.2)$ \\
CAR (mean (SD)) & 2 & $22(21.0)$ \\
NLR (mean (SD)) & 3 & $9(8.6)$ \\
PLR (mean (SD)) & 4 & $6(5.7)$ \\
Pathological Stage & 5 & $2(1.9)$ \\
& 6 & $1(1.0)$ \\
& & $47.55(5.11)$ \\
& & $0.121(0.213)$ \\
& & $2.71(1.64)$ \\
& II & $159.40(72.08)$ \\
& III & $27(25.7)$ \\
& IV & $25(23.8)$ \\
& & $40(38.1)$ \\
& & $13(12.4)$ \\
\hline
\end{tabular}

SD: Standard deviation; mGPS: modified Glasgow prognostic score CONUT: controlling nutritional status; PNI: prognostic nutritional index; CAR: C-reactive protein-to-albumin ratio; NLR: neutrophil-to-lymphocyte ratio; PLR: platelet-to-lymphocyte ratio.

for continuous variables. A correlation analysis was performed using Spearman's rank coefficients. The Jonckheere-Terpstra test was used for trend analysis. Univariate and multivariate survival analyses were carried out using the Cox proportional hazards regression model. Kaplan-Meier curves were generated for OS, and significance was assessed using the log-rank test. Receiver operating characteristics (ROC) curves were generated, and areas under the curve (AUC) were used to evaluate the discriminatory ability of the CONUT score, PNI, CAR, NLR, and PLR to predict OS. A probability value of $p<0.05$ was considered significant. All analyses were performed using the R version 2.13.0 (The R Foundation for Statistical Computing, Vienna, Austria) statistical software.

\section{Results}

Patient characteristics. The flowchart presented in Figure 1 outlines this study. In total, 177 patients were assessed for eligibility to be included in the study. Subsequently, seventytwo patients were excluded. One patient was excluded for receiving maintenance hemodialysis, 33 patients for undergoing preoperative therapy, 38 patients for having no data of neutrophil, lymphocyte, platelet, CRP, albumin, or cholesterol measurements in peripheral blood within one month before surgery. The remaining 105 patients were
Table II. Univariate and multivariate analyses for overall survival.

\begin{tabular}{|c|c|c|c|c|}
\hline \multirow[b]{2}{*}{$\begin{array}{l}\text { Characteristics } \\
\text { Value }\end{array}$} & \multicolumn{2}{|l|}{ Univariate } & \multicolumn{2}{|c|}{ Multivariate } \\
\hline & HR $(95 \% \mathrm{CI})$ & $p$-Value & HR $(95 \% \mathrm{CI})$ & $p$ \\
\hline Age (years) & $1.01(0.97-1.05)$ & 0.78 & $0.99(0.95-1.03)$ & 0.69 \\
\hline \multicolumn{5}{|l|}{ Gender } \\
\hline $\begin{array}{l}\text { Female } \\
\text { (reference) }\end{array}$ & 1 & 0.220 & 1 & 0.110 \\
\hline Male & $2.47(0.59-10.37)$ & & $3.26(0.77-13.81)$ & \\
\hline \multicolumn{5}{|l|}{ mGPS } \\
\hline 0 (reference) & 1 & 0.092 & & \\
\hline 1,2 & $2.06(0.89-4.8)$ & & & \\
\hline \multicolumn{5}{|l|}{ CONUT score } \\
\hline$<3$ (reference) & 1 & 0.420 & & \\
\hline$\geq 4$ & $1.54(0.54-4.41)$ & & & \\
\hline \multicolumn{5}{|c|}{ (10) } \\
\hline $\begin{array}{l}<40.2 \\
\quad \text { (reference) }\end{array}$ & 1 & 0.210 & & \\
\hline$\geq 40.2$ & $0.51(0.18-1.47)$ & & & \\
\hline \multicolumn{5}{|l|}{ CAR } \\
\hline $\begin{array}{l}<0.026 \\
\text { (reference) }\end{array}$ & 1 & 0.001 & 1 & 0.017 \\
\hline$\geq 0.026$ & $3.60(1.66-7.82)$ & & $2.69(1.19-6.06)$ & \\
\hline \multicolumn{5}{|l|}{ NLR } \\
\hline $\begin{array}{l}<1.594 \\
\text { (reference) }\end{array}$ & 1 & 0.310 & & \\
\hline$\geq 1.594$ & $0.68(0.32-1.44)$ & & & \\
\hline \multicolumn{5}{|l|}{ PLR } \\
\hline $\begin{array}{l}<164.6 \\
\quad(\text { reference) }\end{array}$ & 1 & 0.058 & & \\
\hline$\geq 164.6$ & $1.98(0.98-4.01)$ & & & \\
\hline \multicolumn{5}{|c|}{ Pathological Stage } \\
\hline I, II (reference) & 1 & & 1 & \\
\hline III, IV & $3.41(1.56-7.47)$ & 0.002 & $2.79(1.21-6.42)$ & 0.016 \\
\hline
\end{tabular}

HR: Hazard ratio; CI: Confidence Interval; mGPS: modified Glasgow prognostic score; CONUT: controlling nutritional status; PNI: prognostic nutritional index; CAR: C-reactive protein-to-albumin ratio; NLR: neutrophil-to-lymphocyte ratio; PLR: platelet-to-lymphocyte ratio.

included in this study. Their baseline characteristics are summarized in Table I.

Cut-off value of prognostic scores. The best cut-off value of the CONUT score, PNI, CAR, NLR, and PLR for OS based on ROC curves were score 4 [AUC 0.501 (95\% confidence interval $(\mathrm{CI})=0.384-0.619)$ ], 40.2 [AUC $0.535(95 \% \mathrm{CI}=0.410$ $0.659)$ ], 0.026 [AUC $0.713(95 \% \mathrm{CI}=0.609=0.818)$ ], 1.594 [AUC $0.499(95 \% \mathrm{CI}=0.365-0.633)$ ], and 164.6 [AUC 0.575 $(95 \% \mathrm{CI}=0.451-0.699)]$, respectively. Concerning mGPS, as the majority of patients $(86.7 \%)$ had a score of 0 , we set the cutoff value as score 1 .

Survival analysis. Univariate analysis revealed CAR and pathological stage to be significant prognostic factors for OS. 


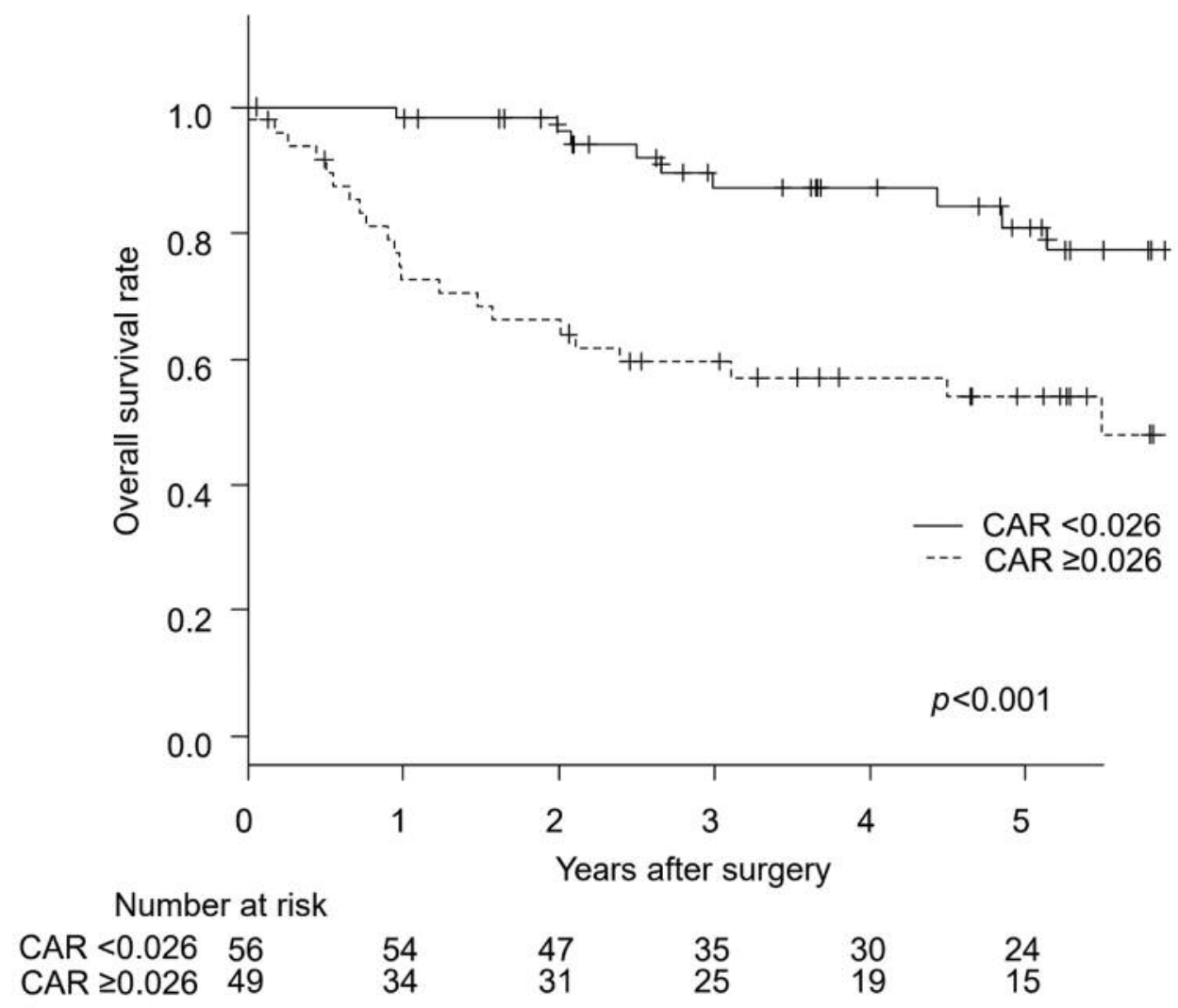

Figure 2. Kaplan-Meier curves for overall survival according to CAR. CAR: C-reactive protein-to-albumin ratio.

Multivariate analysis demonstrated CAR and pathological stage to be independent prognostic factors for OS (Table II). The OS rate was significantly lower for patients with $\mathrm{CAR} \geq 0.026$ compared to those with $\mathrm{CAR}<0.026$ ( $p<0.001$; Figure 2$)$.

Relationship between CAR and clinicopathological variables. The relationships between CAR and clinicopathological variables are shown in Table III. CAR $\geq 0.026$ was associated with more advanced pathological TNM stage $(p=0.001)$. CAR as a continuous variable tended to increase as the TNM stage progressed $(p$ for trends $=0.005$ ) (Figure 3$)$.

\section{Discussion}

The most important finding of the present study is that the preoperative CAR was associated with a poor prognosis and an independent prognostic factor for the OS of EC patients who underwent McKeown esophagectomy with gastric tube reconstruction without neoadjuvant therapy. We also confirmed the tumor-stage related increase in CAR as both a subject as well as a continuous variable. This suggested that this association was independent of the cut-off value, and that a high CAR is associated with tumor progression in EC patients.
Table III. Patient characteristics by CAR.

\begin{tabular}{lccc}
\hline & \multicolumn{3}{c}{ CAR } \\
\cline { 2 - 4 } Characteristics & $\begin{array}{c}<0.026 \\
(\mathrm{n}=56)\end{array}$ & $\begin{array}{c}\geq 0.026 \\
(\mathrm{n}=49)\end{array}$ & $p$-Value \\
\hline Age (years) [mean (SD)] & $63.98(8.55)$ & $65.63(8.93)$ & 0.336 \\
Gender & $49(87.5)$ & $44(89.8)$ & 0.767 \\
$\quad$ Male & $7(12.5)$ & $5(10.2)$ & \\
$\quad$ Female & $7(12.5)$ & $4(8.2)$ & 0.608 \\
Location of tumor & $29(51.8)$ & $23(46.9)$ & \\
$\quad$ Upper & $20(35.7)$ & $22(44.9)$ & \\
$\quad$ Middle & & & \\
$\quad$ Lower & $36(64.3)$ & $16(32.7)$ & 0.002 \\
Pathological Stage & $20(35.7)$ & $33(67.3)$ & \\
$\quad$ I, II & &
\end{tabular}

SD: Standard deviation; CAR: C-reactive protein-to-albumin ratio.

We found CAR to be an independent prognostic factor for the OS of EC patients who underwent surgery, which is consistent with previous reports $(4,5)$. These findings suggest that CAR can identify the subgroup of patients who 


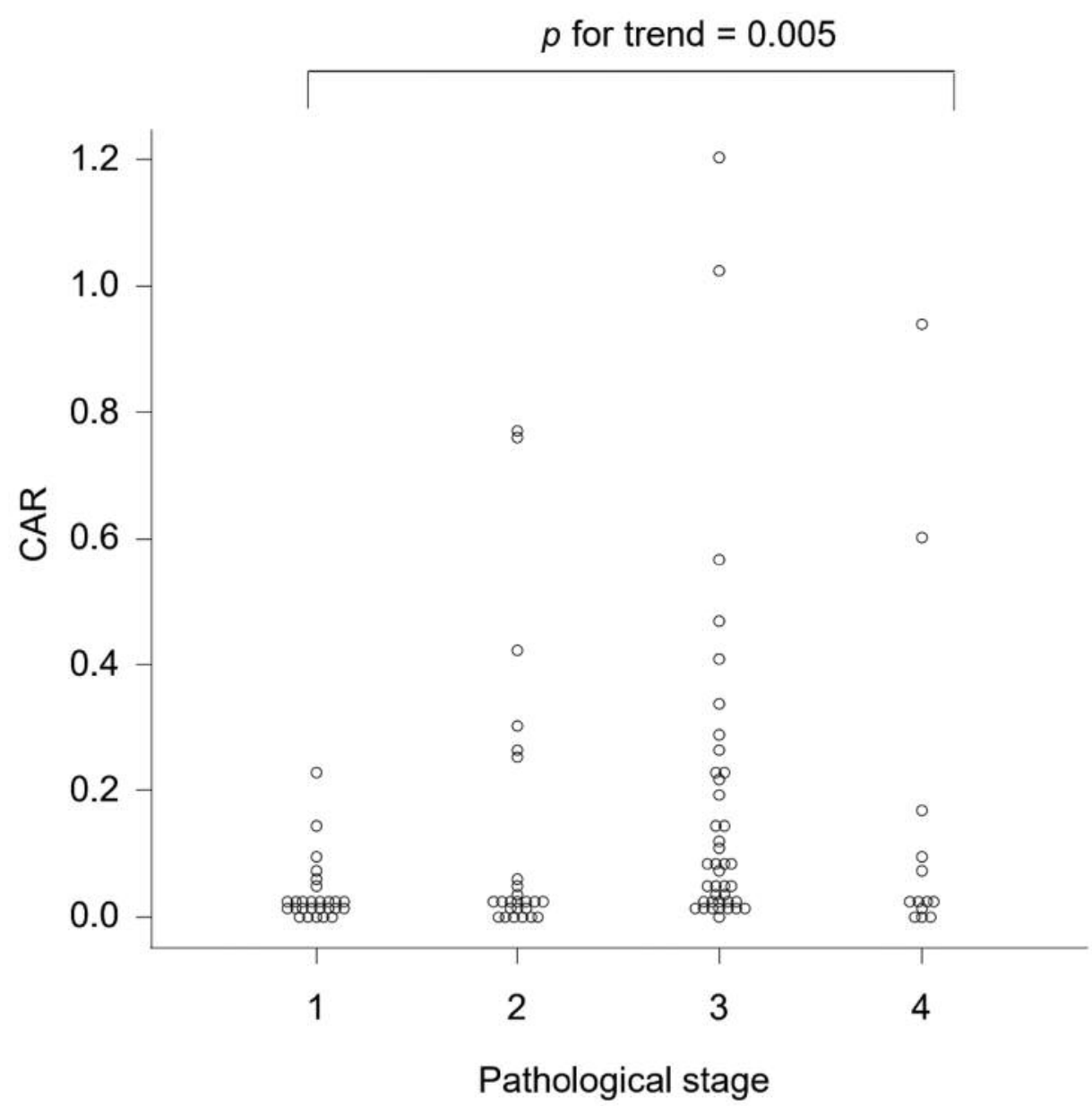

Figure 3. Association between CAR and pathological stage. CAR: C-reactive protein-to-albumin ratio.

can be at risk of poor prognosis when undergoing surgical resection independently of pathological staging.

Hepatocytes produce CRP as a systemic response to IL-6 released from leukocytes within the tumor microenvironment (16). IL-6 promotes inflammatory cytokine production, tumor angiogenesis, and tumor growth in esophageal cancer cell lines (17). CRP significantly increases in cancer patients with higher serum levels of IL-6 (18), which in turn are also correlated with lower serum levels of albumin in EC patients (18). Furthermore, CRP has been reported to correlate with the depth of invasion, the degree of lymph node metastasis, and the presence of distant metastasis (19). The above associations among CRP, IL-6, and albumin are possible explanations for the malignant potential of the tumors with a high CAR.

In our study, mGPS, CONUT score, PNI, NLR, and PLR were not independent prognostic factors. Regarding mGPS and CONUT, the majority of patients had an mGPS of score $0(86.7 \%)$ and a normal to mild nutritional status (CONUT score 0-4) (97.1\%). Thus, patients with abnormal mGPS and CONUT values were the minority in our cohort, and it was not possible to distinguish the survival difference of patients in our study based on these values. Furthermore, based on the CONUT score, most of the patients had no severe malnutrition in our study, explaining why a cohort of our patients with a poorer prognosis was unable to be identified using PNI.

A high CRP level is known to be a response to secondary tumor necrosis and damage (16). Systemic chemotherapy or radiation has been reported to significantly impact systemic inflammation $(20,21)$. Therefore, measurement of inflammation-based prognostic parameters in patients receiving neoadjuvant treatments may not accurately reflect the baseline impact of systemic inflammation on survival. In EC cancer, changes during chemotherapy in the mGPS, which consists of albumin and CRP, have been reported to affect the prognosis of patients (22). The preoperative treatment has a unignorable impact on the prognosis, and 
precise pathological staging in the resected specimens was vital for the investigation of prognostic factors. In this context, our study, focusing on patients who underwent the same surgical procedure (McKeown esophagectomy with gastric tube reconstruction) without preoperative therapy, evaluated the prognostic significance of CAR in EC patients more precisely. On the other hand, this study had several limitations. First, this was a retrospective single-institution analysis. Second, the cut-off values of the CONUT score, PNI, CAR, NLR, and PLR may have been biased because they were selected using ROC curves.

In conclusion, preoperative CAR was associated with a poor prognosis and was an independent prognostic factor for the OS of EC patients who underwent McKeown esophagectomy with gastric tube reconstruction without neoadjuvant therapy. The tumor-stage related increase in CAR as both a subject and a continuous variable suggests that a high CAR is associated with tumor progression in EC patients.

\section{Conflicts of Interest}

The Authors have no conflicts of interest to declare.

\section{Authors' Contributions}

Study conception and design was performed by MS; acquisition of data by $\mathrm{HS}, \mathrm{YU}, \mathrm{NN}, \mathrm{KK}, \mathrm{KH}$; analysis and interpretation of data was done by MS, MSo, AS, KO, TY; drafting of the manuscript by MS; critical revision by KS and HS. All Authors read and approved the final version of the manuscript.

\section{References}

1 Yu X, Wen Y, Lin Y, Zhang X, Chen Y, Wang W, Wang G and Zhang L: The value of preoperative Glasgow Prognostic Score and the C-Reactive Protein to Albumin Ratio as prognostic factors for long-term survival in pathological T1N0 esophageal squamous cell carcinoma. J Cancer 9: 807-815, 2018. PMID: 29581759. DOI: $10.7150 /$ jca.22755

2 Hirahara N, Matsubara T, Hayashi H, Takai K, Nakada S and Tajima Y: Prognostic importance of controlling nutritional status in patients undergoing curative thoracoscopic esophagectomy for esophageal cancer. Am J Ther 25: e524-e532, 2018. PMID: 26866437. DOI: 10.1097/MJT.0000000000000414

3 Nozoe T, Kimura Y, Ishida M, Saeki H, Korenaga D and Sugimachi K: Correlation of pre-operative nutritional condition with post-operative complications in surgical treatment for oesophageal carcinoma. Eur J Surg Oncol 28: 396-400, 2002. PMID: 12099649. DOI: 10.1053/ejso.2002.1257

4 Ishibashi Y, Tsujimoto H, Hiraki S, Kumano I, Yaguchi Y, Horiguchi H, Nomura S, Ito N, Shinto E, Aosasa S, Yamamoto $\mathrm{J}$ and Ueno H: Prognostic value of preoperative systemic immunoinflammatory measures in patients with esophageal cancer. Ann Surg Oncol 25: 3288-3299, 2018. PMID: 30019304. DOI: $10.1245 / \mathrm{s} 10434-018-6651-\mathrm{y}$

5 Kunizaki M, Tominaga T, Wakata K, Miyazaki T, Matsumoto K, Sumida Y, Hidaka S, Yamasaki T, Yasutake T, Sawai T,
Hamamoto R, Nanashima A and Nagayasu T: Clinical significance of the $\mathrm{C}$-reactive protein-to-albumin ratio for the prognosis of patients with esophageal squamous cell carcinoma. Mol Clin Oncol 8: 370-374, 2018. PMID: 29435305. DOI: $10.3892 / \mathrm{mco} .2017 .1527$

6 Liu X, Sun X, Liu J, Kong P, Chen S, Zhan Y and Xu D: Preoperative C-reactive protein/albumin ratio predicts prognosis of patients after curative resection for gastric cancer. Transl Oncol 8: 339-345, 2015. PMID: 26310380. DOI: 10.1016/ j.tranon.2015.06.006

7 Jomrich G, Paireder M, Gleiss A, Kristo I, Harpain L and Schoppmann SF: Comparison of inflammation-based prognostic scores in a cohort of patients with resectable esophageal cancer. Gastroenterol Res Pract 2017: 1-10, 2017. PMID: 28740506. DOI: $10.1155 / 2017 / 1678584$

8 Zhang X, Jiang Y, Wang Y, Wang Z, Zhao L, Xue X, Sang S and Zhang L: Prognostic role of neutrophil-lymphocyte ratio in esophageal cancer. Medicine (Baltimore) 97: e13585, 2018. PMID: 30544482. DOI: 10.1097/MD.0000000000013585

9 Feng J-F, Huang Y and Chen Q-X: Preoperative platelet lymphocyte ratio (PLR) is superior to neutrophil lymphocyte ratio (NLR) as a predictive factor in patients with esophageal squamous cell carcinoma. World J Surg Oncol 12: 58, 2014. PMID: 24641770. DOI: 10.1186/1477-7819-12-58

10 Rusch VW, Rice TW, Crowley J, Blackstone EH, Rami-Porta R and Goldstraw P: The seventh edition of the American Joint Committee on Cancer/International Union Against Cancer Staging Manuals: The new era of data-driven revisions. J Thorac Cardiovasc Surg 139: 819-821, 2010. PMID: 20304130. DOI: 10.1016/j.jtcvs.2010.02.013

11 McMillan DC, Crozier JEM, Canna K, Angerson WJ and McArdle CS: Evaluation of an inflammation-based prognostic score (GPS) in patients undergoing resection for colon and rectal cancer. Int J Colorectal Dis 22: 881-886, 2007. PMID: 17245566. DOI: $10.1007 / \mathrm{s} 00384-006-0259-6$

12 Ignacio de Ulíbarri J, González-Madroño A, de Villar NGP, González P, González B, Mancha A, Rodríguez F and Fernández G: CONUT: a tool for controlling nutritional status. First validation in a hospital population. Nutr Hosp 20: 38-45. PMID: 15762418.

13 Harimoto N, Yoshizumi T, Sakata K, Nagatsu A, Motomura T, Itoh S, Harada N, Ikegami T, Uchiyama H, Soejima Y and Maehara Y: Prognostic significance of preoperative controlling nutritional status (CONUT) score in patients undergoing hepatic resection for hepatocellular carcinoma. World J Surg 41: 2805-2812, 2017. PMID: 28653142. DOI: 10.1007/s00268017-4097-1

14 Onodera T, Goseki N and Kosaki G: Prognostic nutritional index in gastrointestinal surgery of malnourished cancer patients. Nihon Geka Gakkai Zasshi 85: 1001-1005, 1984. PMID: 6438478

15 Miyazaki T, Yamasaki N, Tsuchiya T, Matsumoto K, Kunizaki M, Kamohara R, Hatachi G, Doi R, Obata T and Nagayasu T: Ratio of C-reactive protein to albumin is a prognostic factor for operable non-small-cell lung cancer in elderly patients. Surg Today 47: 836-843, 2017. PMID: 27853867. DOI: 10.1007/ s00595-016-1448-8

16 Wang C-S and Sun C-F: C-reactive protein and malignancy: clinico-pathological association and therapeutic implication. Chang Gung Med J 32: 471-82. PMID: 19840504. 
17 Zhao Z-F, Li J-X, Ye R, Wu X, Gao L-L and Niu B-L: Interleukin- 6 as a potential molecular target in esophageal squamous cell carcinoma. Oncol Lett 11: 925-932, 2016. PMID: 26893670. DOI: 10.3892/ol.2015.3990

18 Oka M, Yamamoto K, Takahashi M, Hakozaki M, Abe T, Iizuka N, Hazama S, Hirazawa K, Hayashi H, Tangoku A, Hirose K, Ishihara $\mathrm{T}$ and Suzuki T: Relationship between serum levels of interleukin 6, various disease parameters and malnutrition in patients with esophageal squamous cell carcinoma. Cancer Res 56: 2776-2780, 1996. PMID: 8665513.

19 Shimada H, Nabeya Y, Okazumi SI, Matsubara H, Shiratori T, Aoki T, Sugaya M, Miyazawa Y, Hayashi H, Miyazaki SI and Ochiai T: Elevation of preoperative serum C-reactive protein level is related to poor prognosis in esophageal squamous cell carcinoma. J Surg Oncol 83: 248-252, 2003. PMID: 12884238. DOI: $10.1002 /$ jso. 10275

20 Namikawa T, Munekage E, Munekage M, Maeda H, Yatabe T, Kitagawa $\mathrm{H}$, Kobayashi $\mathrm{M}$ and Hanazaki $\mathrm{K}$ : Evaluation of systemic inflammatory response biomarkers in patients receiving chemotherapy for unresectable and recurrent advanced gastric cancer. Oncology 90: 321-326, 2016. PMID: 27225990. DOI: $10.1159 / 000446373$
21 Badakhshi H, Kaul D and Zhao K-L: Association between the inflammatory biomarker, C-reactive protein, and the response to radiochemotherapy in patients with esophageal cancer. Mol Clin Oncol 4: 643-647, 2016. PMID: 27073683. DOI: 10.3892/ mco. 2016.753

22 Otowa Y, Nakamura T, Takiguchi G, Tomono A, Yamamoto M, Kanaji S, Imanishi T, Suzuki S, Tanaka K, Itoh T and Kakeji Y: Changes in modified Glasgow prognostic score after neoadjuvant chemotherapy is a prognostic factor in clinical stage II/III esophageal cancer. Dis Esophagus 29: 146-151, 2016. PMID: 25515972. DOI: 10.1111/dote.12316

Received October 22, 2019

Revised November 29, 2019

Accepted December 2, 2019 